\title{
Microwave Irradiation Synthesis and Characterization of Silver Doped Bismuth Oxyiodide Microflowers with Enhanced Daylight Photocatalytic Performance
}

\author{
Nuengruethai Ekthammathat, Pornchai Pornharuthai, and Anukorn Phuruangrat
}

\begin{abstract}
Silver doped bismuth oxyiodide (Ag-doped BiOI) microsflowers have been successfully synthesized by a simple microwave irradiation method. The optimum condition was synthesized with $\mathrm{pH} 3$ at $180 \mathrm{~W}$ for $3 \mathrm{~h}$. The contents of $\mathrm{Ag}$ dopant were in the range $0.0-3.0 \mathrm{~mol} \%$. The phase purity and crystallinity of the product were characterized by XRD technique. It indicated that the products are pure tetragonal BiOI phase. The morphologies of series Ag-,doped BiOI were microflowers, diameter about 700-1,000 $\mu \mathrm{m}$ which investigated by SEM and TEM technique. Then, 0.0-3.0 mol\% Ag-doped BiOI were studied the photocatalytic properties by degradation of methylene blue (MB) solution under daylight for $100 \mathrm{~min}$. The decolorization efficiency (\%) of $0.0,0.5,1.0,2.0$, and 3.0 mol\% Ag-doped were 81.89, 96.46, 96.8, 92.43, and $90.59 \%$, respectively. So, it can be concluded that $\mathrm{Ag}^{+}$ion has been prevented electro-hole recombination, the photocatalytic property was improved. The research has many advantages about synthesized Ag-doped BiOI by a simply, fast, and low cost method. Moreover, as-synthesized Ag-doped BiOI can apply in waste water treatment, degradation of organic pollutant and so on.
\end{abstract}

Index Terms—BiOI, silver doped, photocatalytic.

\section{INTRODUCTION}

Technologies of catalyst are critical to present and future energy, chemical process, and environmental, as widely recognized and concerned [1]. Especially, it has been interested as photocatalyst which based on the promotion of oxidation - reduction reactions by photoexcited electron and holes from semiconductor under light irradiation or photon energy [2], [3]. Therefore, this is the one of the ways to degrade the organic pollution by the oxidation of organic pollutant into $\mathrm{CO}_{2}, \mathrm{H}_{2} \mathrm{O}$, inorganic ions and non-toxic molecules as environmental remediation [2]. The traditional photocatalyst, such as $\mathrm{TiO}_{2}, \mathrm{ZnO}$, etc., were widely studied but they are active only under ultraviolet light region (which corresponds to 4-5\% of incident sun light [2], [4], [5]) and

Manuscript received January 25, 2017; revised May 20, 2017.This work was supported by Bansomdejchaopraya Rajabhat University (BSRU) for financial support and Science and Appiled Science Center (SASC), faculty of science and technology for facility support.

N. Ekthammathat is with the Program of Chemistry, Faculty of Science and Technology, Bansomdejchaopraya Rajabhat University, Bangkok 10600 Thailand (corresponding e-mail: nueng_omam@hotmail.com).

P. Pornharuthai is with the Program of Tool and Die Engineering, Faculty of science and Technology, Bansomdejchaophraya Rajabhat University, Bangkok 10600, Thailand (e-mail: lava_pp10@hotmail.com).

A. Phuruangrat is with the Department of Materials Science and Technology, Faculty of Science, Prince of Songkla University, Hat Yai, Songkhla 90112, Thailand (e-mail: phuruangrat@hotmail.com). high recombination rates of photoinduced electron-hole pairs, rendering them unsuitable for practical applications [4], [6].

So, the researchers have been developed the photocatalytic properties of the materials to active under visible light region (which corresponds to 43-45\% of incident sun light [4], [5]) by many technique such as ion/element doping, composite, or finding the noble materials. There are many reported about a new photocatalyst which high performance for instance $\mathrm{CuO}$ [7], $\mathrm{MgO}$ [8], $\mathrm{CeVO}_{4}$ [9], $\mathrm{Bi}_{2} \mathrm{WO}_{6}$ [10], $\mathrm{BiVO}_{4}$ [11], $\mathrm{BiOX}$ $(\mathrm{X}=\mathrm{Cl}, \mathrm{Br}, \mathrm{I})$ [12], and so on. One of them, p-type semiconductor bismusth oxyiodide (BiOI) has been attention because it has narrow band gap energy about $1.77 \mathrm{eV} \mathrm{[4],}$ [13], [15] thereby it has been active under visible light region.

In addition, $\mathrm{BiOI}$ has a unique structure of alternate stacking of tetragonal layered structure containing $\left[\mathrm{Bi}_{2} \mathrm{O}_{2}\right]^{2+}$ slabs with the I-slabs (double slabs of halogen atom [4]). Owing to strong intralayer bonding and weak interlayer van der Waals interaction [16] corresponded photo-generated holes and electrons in BiOI can be efficiently separated [4], [6], [13], [15], [16]. So, BiOI has a high performance photocatalytic activity and widely application such as water decontamination [13], [15], hazardous removal [17], anti-bacterial [13], $\mathrm{H}_{2}$ production [17], and etc.

Moreover, ion/metal doping as $\mathrm{Pt}, \mathrm{Ag}$, $\mathrm{Au}$ in to the structure or on the surface of catalyst also displayed remarkable activity improvement. Due to the defect energy levels are introduced to capture electrons and crystallization can be changed which prevent the recombination probability of light-induce electrons and holes, formation of a Schottky barrier. So this strategy can improved the photocatalytic activity and also shifts the absorption edge to visible light region by reducing band gap energy of catalysts, plasmonic effect [18], [19].

Thus, this research was synthesis $\mathrm{BiOI}$ microstructures by microwave irradiation method and improved the properties by Ag-doping strategy. This method has many advantages such as simply, fast, low temperature, and low cost. Then, the products were characterized by XRD, SEM, TEM, and studies the photocatalytic activities by decolorization of methylene blue under visible light region.

\section{Procedure OR EXPERIMENT}

\section{A. Synthesis}

All the reagents of this research are analytical grade and use without further purification. BiOI (undoped Ag) was 
synthesized from bismuth nitrate $\left(\mathrm{Bi}\left(\mathrm{NO}_{3}\right)_{3} \cdot 5 \mathrm{H}_{2} \mathrm{O}\right)$ and potassium iodide $(\mathrm{KI})$ as starting materials in a $1: 1$ ratio, dissolved in $150 \mathrm{ml} \mathrm{DI}$ water. The $\mathrm{pH}$ of precursor solutions was adjusted to pH 3 by $3 \mathrm{M} \mathrm{NaOH}$, stirred for 15 min at room temperature. Subsequently, the solution was microwave irradiation processed at $180 \mathrm{~W}$ for $3 \mathrm{~h}$. At last, the precipitates were filtrated, wash with DI water and ethanol, and dried at $80{ }^{\circ} \mathrm{C}$ for $24 \mathrm{~h}$.

Another one, the series of Ag-doped BiOI was synthesized by similar method. It has been added $0.5-3 \mathrm{~mol} \%$ of $\mathrm{AgNO}_{3}$ into solution, together with starting materials adjusted the $\mathrm{pH}$ to 3 by $3 \mathrm{M} \mathrm{NaOH}$, stirred for $15 \mathrm{~min}$ at room temperature. Next, the solutions was microwave irradiation processed at $180 \mathrm{~W}$ for $3 \mathrm{~h}$, filtrated and wash the precipitation with DI water and ethanol, and dried at $80{ }^{\circ} \mathrm{C}$ for $24 \mathrm{~h}$.

\section{B. Characterization}

The crystallinity and phases identification of the products were analyzed by the X-ray diffractometer (XRD, Philips $\mathrm{X}^{\prime}$ Pert MPD) using a $\mathrm{CuK}_{\alpha}$ radiation at $45 \mathrm{kV}$ and $35 \mathrm{~mA}$ in the range of 25-60 deg. The size and morphology of as-synthesized Ag-doped BiOI was studied by Low vacuum scanning electron microscope (LV-SEM, JEOL IT300) operating at $20 \mathrm{kV}$. Transmission electron microscopic images and corresponding selected area electron diffraction (SAED) pattern of the as-prepared Ag-doped BiOI sample were taken with a transmission electron microscope (TEM, JEOL, JEM-2100F) at $200 \mathrm{kV}$.

\section{Photocatalytic Activity Test}

Photocatalytic activities of the products were studies by decolorization of methylene blue (MB) in an aqueous solution under daylight. The photoreactor used light source as Philips Lifemax 32W 6200K Cool Daylight. In a typical degradation experiment, $100 \mathrm{ml} \mathrm{MB}$ solution with a concentration 10-5 $\mathrm{M}$ and $100 \mathrm{mg}$ as-prepared Ag-doped BiOI were added into a $250 \mathrm{ml}$ Pyrex Erlenmeyer flask, and then stirred continuously in the dark for $30 \mathrm{~min}$ to ensure adsorption/desorption equilibrium of $\mathrm{MB}$ on the surfaces of the photocatalyst. The equilibrium concentration of MB was used as the initial value for the photodegradation processes. The light was turned on to initiate the photocatalytic reaction. At certain time intervals, $5 \mathrm{ml}$ solution were sampled and centrifuged to remove the particles. The degradation of organic dye was monitored by measuring the absorbance of the solution using the UV-visible spectrophotometer with DI water as reference and measure at wavelength of $665 \mathrm{~nm}$.

\section{ReSUlts AND DisCUSSION}

The phase purity and crystallinity of as-synthesized Ag-BiOI were investigated by XRD technique, the XRD patterns of 0.0, 0.5, 1.0, 2.0 and 3.0 mol\% Ag-doped BiOI were shown in Fig. 1. They indicated all products were pure tetragonal $\mathrm{BiOI}$ phase with apace group $\mathrm{P} 4 / \mathrm{nmm}$ in accordance with the JCPDS No. 10-0445 [20]. The diffraction peaks at $2 \Theta=29.64,31.65,37.05,37.39,45.37$, 46.48 and 51.34 can be indexed to (102), (110), (103), (112), (200), (201) and (114) crystallographic plan of BiOI phase, respectively. And, the content of 0.0-3.0 mol\% Ag dopants were effected to the crystallinity of $\mathrm{BiOI}$ products. According to the intensities and sharp of XRD spectra were decreased after increased the content of Ag from 0.0 to $3.0 \mathrm{~mol} \% \mathrm{Ag}$. In addition, the metallic Ag phase was not detected. In this case, it means $\mathrm{AgNO}_{3}$ which added into the reaction was released $\mathrm{Ag}+$ and inserted in crystal structure of BiOI as well. The formation mechanism of $\mathrm{BiOI}$ and chemical reaction steps were described as the following equations [17]:

$$
\begin{gathered}
\mathrm{Bi}\left(\mathrm{NO}_{3}\right)_{3}+\mathrm{H}_{2} \mathrm{O} \rightarrow \mathrm{BiONO}_{3}+2 \mathrm{HNO}_{3} \\
\mathrm{BiONO}_{3}+\mathrm{KI} \rightarrow \mathrm{BiOI}+\mathrm{KNO}_{3} \\
\mathrm{BiOI}+\mathrm{AgNO}_{3} \rightarrow \text { Ag-doped BiOI }
\end{gathered}
$$

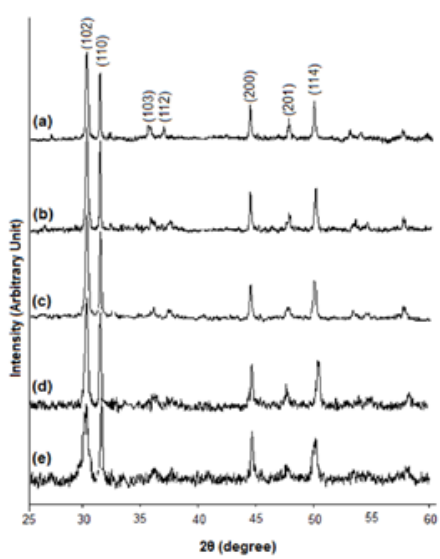

Fig. 1. XRD patterns of (a) 0.0, (b) 0.5, (c) 1.0, (d) 2.0, and (e) $3.0 \mathrm{~mol} \%$ Ag-doped BiOI which synthesized by microwave irradiation method with $180 \mathrm{~W} 3 \mathrm{~h}$.

Fig. 2 show the SEM images of series with $0.0-3.0 \mathrm{~mol} \%$ Ag-doped BiOI at magnification about 40,000 times. The morphologies of the products are microflowers in the range size of $700-1000 \mu \mathrm{m}$. The microflowers composed of many plates with smooth surface. At $0.0 \%$ Ag-doped $\mathrm{BiOI}$ is the biggest size (Fig. 2a). When the contents of Ag were increased, 0.0 to $2.0 \mathrm{~mol} \% \mathrm{Ag}$, the size of product were decreased. But, at $3.0 \mathrm{~mol} \% \mathrm{Ag}$ doped, the size of products become to big size again (Fig. 4a). So, the smallest size is 2.0 mol\% Ag doped BiOI. Then, we analyses $\mathrm{Ag}+$ in the structure by EDS technique, the results as shows in Fig. 3. The EDS results show as-synthesized Ag-doped BiOI have Ag in structures, which cannot detected by XRD technique. Moreover, the content of \% mol Ag not equivalent to AgNO3 or $\mathrm{Ag}^{+}$ions added because some $\mathrm{Ag}^{+}$ion loss in reaction. Therefore, the 1.0, 2.0, and $3.0 \%$ mol Ag doped BiOI were detected about $0.25,0.46$, and 1.22 , respectively (insert table, Fig. 3), by EDS technique. So, the losses of \% mol Ag were significantly.

TEM technique was used to analysis of phase and morphology of as-synthesized. TEM image (Fig. 4a) and selected area electron diffraction (SAED) pattern (Fig. 4b) of undoped BiOI were show in Fig. 4. The as-synthesized undoped BiOI remain as clusters of nanoplates, composing the microflowers. The SAED pattern indicated that the product was the single crystalline BiOI, indexed to (200), (220), (020), and (-220) plane with the electron beam along the [00-1] direction of the as-synthesized BiOI, corresponded to XRD result. 


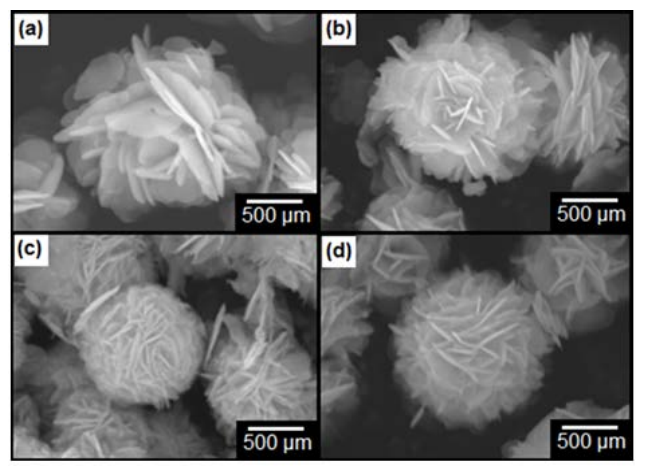

Fig. 2. SEM images of (a) 0.0, (b) 1.0, (c) 2.0, and (d) 3.0 mol\% Ag-doped $\mathrm{BiOI}$ which synthesized by microwave irradiation method with $180 \mathrm{~W} 3 \mathrm{~h}$.

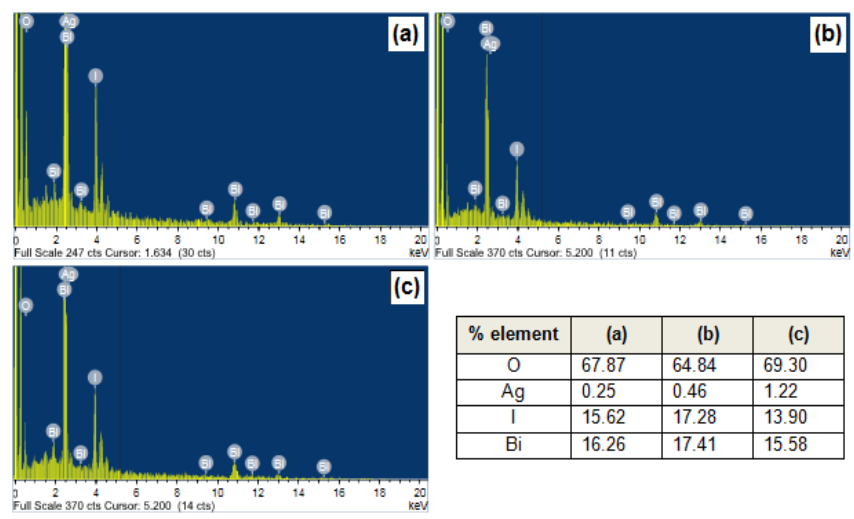

Fig. 3. EDS results of (a) 1.0, (b) 2.0, and (c) 3.0 mol\% Ag-doped BiOI which synthesized by microwave irradiation method with $180 \mathrm{~W} 3 \mathrm{~h}$.

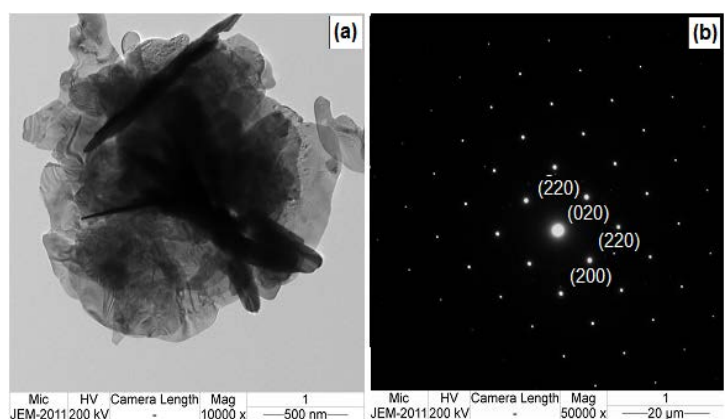

Fig. 4. TEM images of undoped BiOI which synthesized by microwave irradiation method with $180 \mathrm{~W} 3 \mathrm{~h}$.

The photocatalytic activities of Ag-doped BiOI were evaluated by the degradation of MB under daylight (visible light region) for $100 \mathrm{~min}$ and presented in Fig. 5. It is well known that there are three possible mechanisms for dye photodegradation as a photolysis process, a dye photosensitization process and a photocatalytic process [4]. But this research studied of dye photolysis, in absence of catalyst are not observable. A slight degradation (Fig. 5a) of MB which indicated that MB dyes have high structural stability and the photolysis as well as the dye-sensitive process has been not effected [4]. Then, study the photocatalytic activity of undoped BiOI and 0.5-3.0 mol\% Ag-doped (Fig. 5b-f) under daylight within $100 \mathrm{~min}$. The intensity of strong characteristic adsorption peaks at $665 \mathrm{~nm}$ was continuously decreased within 100 min length of irradiation time, indicating the degradation of $\mathrm{MB}$ by photocatalysis process. It can be seen that the decolorization efficiency (\%) of 0.0, 0.5, 1.0, 2.0, and $3.0 \mathrm{~mol} \%$ Ag-doped are $81.89,96.46,96.80,92.43$, and $90.59 \%$, respectively. It can be concluded that 0.5 and $1.0 \mathrm{~mol} \%$ Ag-doped BiOI have higher 0.0, 2.0 and 3.0 mol\% Ag-doped BiOI, as shown in Fig. 6. So, this case Ag+ ion affected the defect energy of BiOI to prevent the recombination [18], [19], so recombination rate was reduced, compared with mol\% Ag-doped BiOI.
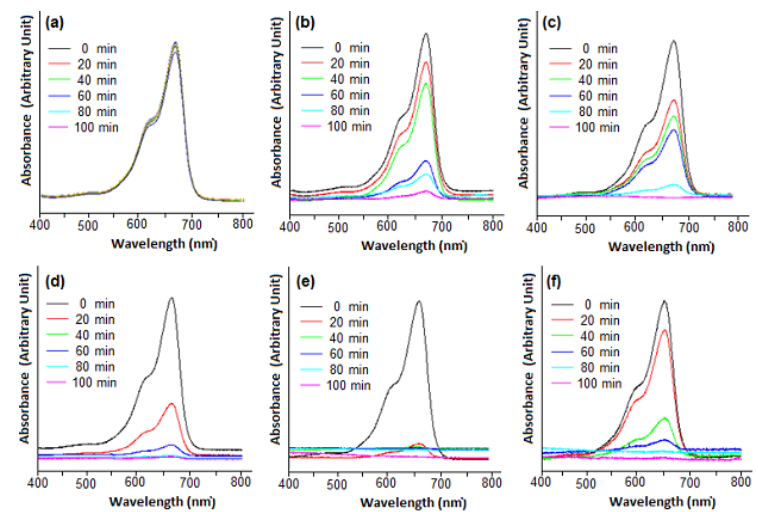

Fig. 5. Absorption spectra of MB solution photocatalyzed by (a) catalyst-free, (b) undoped BiOI, (c) 0.5 mol\% Ag-doped, (d) 1.0 mol\% Ag-doped, (e) 2.0 mol\% Ag-doped, and (f) 3.0 mol\% Ag-doped BiOI under daylight within 100 min.

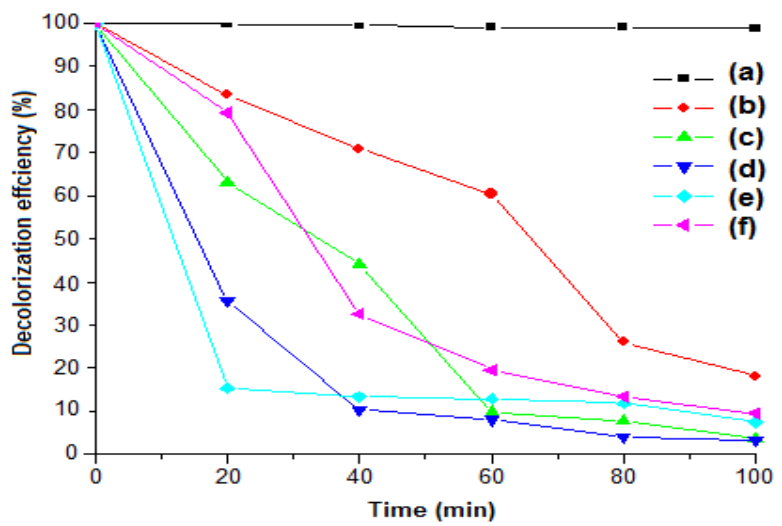

Fig. 6. Decolorization efficiency of the MO solution photocatalyzed by by (a) catalyst-free, (b) undoped BiOI, (c) 0.5 mol\% Ag-doped, (d) $1.0 \mathrm{~mol} \%$ Ag-doped, (e) 2.0 mol\% Ag-doped, and (f) 3.0 mol\% Ag-doped BiOI under daylight within $100 \mathrm{~min}$.

The schematic mechanism of the photocatalytic activity improvement of Ag-doped BiOI is shown in Fig. 7. Normally, photocatalytic process is mainly based the generation and separation of the photoinduced electron-hole pairs. If photoinduced $\mathrm{e}^{-} / \mathrm{h}^{+}$can be separated effectively, it is be benefited to the photocatalytic activities. There is the photocatalytic mechanism under visible light excitation, the electron of BiOI can be promoted from the valence band to the conduction band, leaving behind a hole $\left(\mathrm{h}^{+}\right)$in the valence band. Then the electron transfer to conduction band and react with the adsorbed $\mathrm{O}_{2}$ on the surface of catalyst to form $\cdot \mathrm{O}_{2}{ }^{-}$. The generated $\cdot \mathrm{O}_{2}{ }^{-}$and $\mathrm{h}^{+}$can oxidize the dye or organic pollutant [4], [14], [21]. All possible photocatalytic reactions are summarized in the following equations;

$$
\begin{aligned}
& \mathrm{BiOI} \text { catalyst }+\mathrm{hv} \rightarrow \mathrm{BiOI}\left(\mathrm{e}_{\mathrm{CB}}^{-}+\mathrm{h}^{+}{ }_{\mathrm{VB}}\right) \\
& \mathrm{BiOI}\left(\mathrm{h}_{\mathrm{VB}}^{+}\right)+\mathrm{H}_{2} \mathrm{O} \rightarrow \mathrm{BiOI}+\mathrm{H}++\cdot \mathrm{OH} \\
& \mathrm{BiOI}\left(\mathrm{h}^{+}{ }_{\mathrm{VB}}\right)+\mathrm{OH}^{-} \rightarrow \mathrm{BiOI}+\cdot \mathrm{OH} \\
& \mathrm{BiOI}\left(\mathrm{e}_{\mathrm{CB}}^{-}\right)+\mathrm{O}_{2} \rightarrow \mathrm{BiOI}+\cdot \mathrm{O}_{2}^{-}
\end{aligned}
$$




$$
\begin{aligned}
& 2 \mathrm{BiOI}\left(\mathrm{e}_{\mathrm{CB}}^{-}\right)+\cdot \mathrm{O}_{2}^{-}+2 \mathrm{H}^{+} \rightarrow \cdot \mathrm{OH}+\mathrm{OH}^{-} \\
& \mathrm{O}_{2}^{-}+2 \mathrm{H}^{+} \rightarrow \mathrm{OH}_{2} \\
& \mathrm{BiOI}\left(\mathrm{e}_{\mathrm{CB}}^{-}\right)+\cdot \mathrm{OH}_{2}+\mathrm{H}^{+} \rightarrow \mathrm{BiOI}+\mathrm{H}_{2} \mathrm{O}_{2} \\
& \mathrm{BiOI}\left(\mathrm{e}_{\mathrm{CB}}^{-}\right)+\mathrm{H}_{2} \mathrm{O}_{2} \rightarrow \text { BiOI }+\cdot \mathrm{OH}+\mathrm{OH}- \\
& \text { Dye }+\cdot \mathrm{O}_{2}^{-}(\cdot \mathrm{OH}) \rightarrow \text { Degradation product }
\end{aligned}
$$

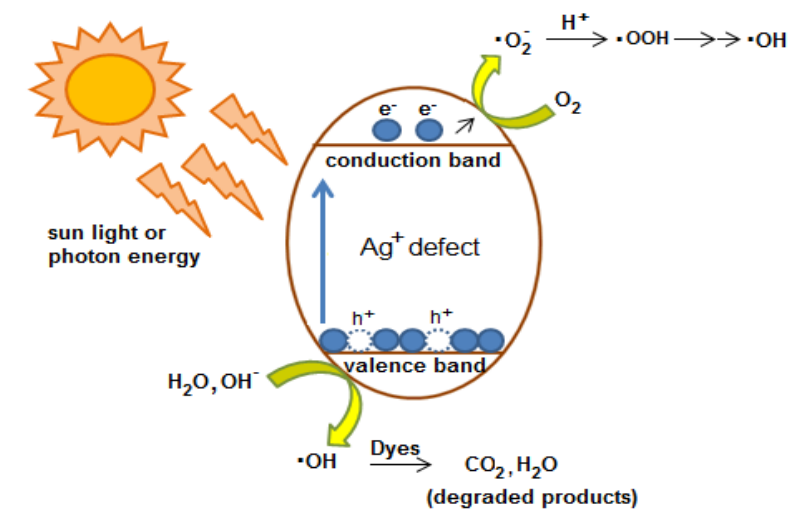

Fig. 7. Schematic mechanism of the photocatalytic activity improvement of Ag-doped BiOI.

Also, the content of ion/metal doped content has been significant influenced the efficiency of electron-hole separation, means Schottky barrier at metal- semiconductor was improved. And it is well known that Ag is cheaper than $\mathrm{Pt}$ and $\mathrm{Au}$. So, this is one of techniques to improve the photocatalytic property of BiOI catalyst.

\section{CONCLUSIONS}

In this study, 0.0-3.0 mol\% Ag-doped BiOI have been successfully synthesized by a simple microwave irradiation method with $\mathrm{pH} 3$ at $180 \mathrm{~W}$ for $3 \mathrm{~h}$. They have an enhancement of photocatalytic performance. Specially, 1.0 mol\% Ag-doped BiOI are the best catalyst because it has the highest percent decolorization efficiency about $96.8 \%$. It can be concluded that $\mathrm{Ag}^{+}$ion has been prevented electro-hole recombination and improved the photocatalytic property of Ag-doped BiOI photocatalyst. The study focuses on synthesized Ag-doped BiOI by microwave irradiation which a simply, fast, and low cost method. Moreover, as-synthesized Ag-doped BiOI can apply in waste water treatment, degradation of organic pollutant and so on.

\section{REFERENCES}

[1] M. M. Khalaf, H. G. Ibrahimov, and E. H. Ismailov, "Nanostructured materials: importance, synthesis and characterizationa review," Chemistry Journal, vol. 2, pp. 118-125, 2012.

[2] R. He, S. Cao, P. Zhou and J. Yu, "Recent advances in visible light Bi-based photocatalysts," Chinese Journal of Catalysis, vol. 35, pp. 989-1007, 2014.

[3] X. Zhang, L. Zhang, T. Xie, and D. Wang, "Low-temperature synthesis and high visible-light-induces photocatalytic activity of $\mathrm{BiOI} / \mathrm{TiO}_{2}$ heterostructures," The Journal of Physical Chemistry C, vol. 113, pp. 7371-7378, 2009.

[4] H. Liu, W. Cao, Y. Su, Y. Wang, and X. Wang, "Synthesis, characterization and photocatalytic performance of novel visible-light-induce Ag/BiOI,” Applied Catalysis B: Environmental, vol. 111-112, pp. 271-279, 2012.
[5] L. Zhu, C. He, Y. Huang, Z. Chen, D. Xia, M. Su, and Y. Xiong, "Enhanced photocatalytic disinfection of E.coli 8099 using Ag/BiOI composite under visible light irradiation," Separation and Purification Technology, vol. 91, pp.59-66, 2009.

[6] M. J. Islam, D. A. Reddy, R. Ma, Y. Kim, and T. K. Kim, "Reduced-graphene-oxide-wrapped BiOI-AgI heterostructured nanocomposite as a high-performance photocatalyst for dye degration under solar light irradiation,” Solid State Sciences, vol. 61, pp. 32-39, 2016.

[7] N. Ekthammathat, T. Thongtem, and S. Thongtem, "Antimicrobial activities of $\mathrm{CuO}$ films deposited on Cu foils by solution chemistry," Applied Surface Science, vol. 277, pp. 211- 217, 2013.

[8] Y. Cai, D. Wu, X. Zhu, W. Wang, F, Tan, J. Chen, X. Qiao, and X. Qiu, "Sol-gel preparation of Ag-doped MgO nanoparticles with high efficiency for bacterial inactivation,” Ceramics International, vol. 43, pp. 1066-1072, 2017.

[9] N. Ekthammathat, A. Phuruangrat, T. Thongtem, and S. Thongtem, "Synthesis and characterization of $\mathrm{CeVO}_{4}$ by microwave radiation method and its photocatalytic activity," Journal of Nanomaterials, vol. 2013, pp.1-7, ID 434197, 2013.

[10] J. Tian, Y. Sang, G. Yu, H. Jiang, X. Mu, and H. Liu, “A Bi ${ }_{2} \mathrm{WO}_{6}$-based hybrid photocatalyst with broad spectrum photocatalytic properties under UV, visible, and near-infrared irradiation,” Advance Materials, vol. 25, pp. 5075-5080, 2013.

[11] S. Zhu, Q. Li, F. Li, W. Cao, and T. Li, "One-pot synthesis of Ag doped $\mathrm{BiVO}_{4}$ microspheres with enhanced photocatalytic activity via a facile hydrothermal method," Journal of Physics and Chemistry of Solids, vol. 92, pp. 11-18.

[12] G. Li, F. Qin, R. Wang, S. Xiao, H. Sun, and R. Chen, "BiOX (X = Cl, $\mathrm{Br}, \mathrm{I})$ nanostructures: mannitol-mediated microwave synthesis, visible light photocatalytic performance, and $\mathrm{Cr}(\mathrm{VI})$ removal capacity," Journal of Colloid and Interface Science, vol. 409, pp. 43-51, 2013.

[13] J. Liang, J. Deng, M. Li, T. Xu, and M. Tong, "Bactericidal activity and mechanism of Ti-doped BiOI microspheresunder visible light irradiation," Colloids and Surfaces B: Biointerfaces, vol. 147, pp. 307-314, 2016.

[14] J. Cao, B. Xu, H. Lin, B. Luo and S. Chen, "Novel heterostructured $\mathrm{Bi}_{2} \mathrm{~S}_{3} / \mathrm{BiOI}$ photocatalyst: facile preparation, characterization and visible light photocatalytic performance,” Dalton Transactions, vol. 41 pp.11482-11490, 2012.

[15] T. Li, S. Luo, and L. Yang, "Three-dimensional hierarchical Ag/AgI/BiOI microspheres with high visible-light photocatalytic activity,” Materials Letters, vol. 109, pp. 247-252, 2013.

[16] L. Ai, Y. Zeng, and J. Jiang, "Hierarchical porous BiOI architectures: facile microwave nonaqueous synthesis, characterization and application in the removal of Congo red from aqueous solution," Chemical Engineering Journal, vol. 235, pp. 331-339, 2014.

[17] C. Zhou, J. Cao, H. Lin, B. Xu, B. Huang, and S. Chen, "Controllable synthesis and photocatalytic activity of $\mathrm{Ag} / \mathrm{BiOI}$ based on themorphology effect of BiOI substrate," Surface and Coatings Technology, vol. 272, pp. 213-220, 2015

[18] J. G. McEvoy and Z. Zhang, "Antimicrobial and photocatalytic disinfection mechanisms in silver-modified photocatalyst under dark and light conditions," Journal of Photochemistry and Photobiology C: Photochemistry Reviews, vol. 19, pp. 62-75, 2014.

[19] S. Zhang, S. Zhang, and L. Song, "Super-high activity of $\mathrm{Bi}^{3+}$ doped $\mathrm{Ag}_{3} \mathrm{PO}_{4}$ and enhanced photocatalytic mechanism," Applied Catalysts B: Environmental, vol. 152-153, pp. 129-139, 2014.

[20] Powder Diffraction File, JCPDS-ICDD, Newtown Square, Pa, USA, 19073-3273, 2001.

[21] N. Ekthammathat, S. Kidarn, A. Phuruangrat, T. Thongtem, and S. Thongtem, "Hydrothermal synthesis of Ag-doped BiOI nanostructure used for photocatalysis," Research Chemical Intermediated, vol. 42, pp. 5559-6672, 2016.

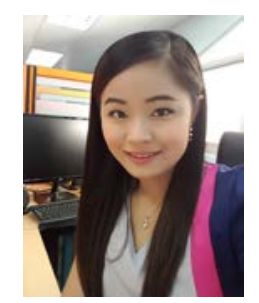

Nuengruethai Ekthammathat was born in Chiangmai, Thailand on November 15, 1986. She finished B.S., M.S., and PhD degree in chemistry in 2009, 2011, and 2014, respectively. Since 2014, she works as a lecturer at Program of Chemistry, Faculty of Science and Technology, Bansomdejchaoproya Rajabhat University (BSRU). In addition, she works at science and applies science center (SASC). Her researches focus on materials science, inorganicchemistry, and photocatalytic. 


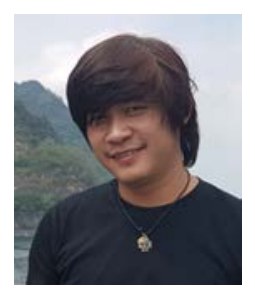

Pornchai Pornharuthai was born in Prathumthani, Thailand on July 5, 1986. He finished B.E. and M.E. degree (electrical engineering) in 2008, and 2012, respectively. Since 2012, he works as a lecturer at Program of Tool and Die Engineering, Faculty of Science and Technology, Bansomdejchaophraya Rajabhat University, Bangkok (BSRU). In addition, he works at renewable energy group, science and apply science center (SASC).

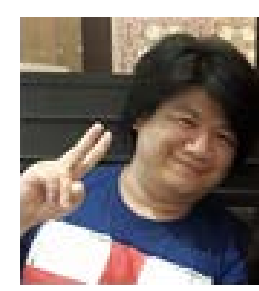

Anukorn Phuruangrat was born in Bangkok, Thailand. Now, he works as a lecturer at the Department of Materials Science and Technology, Faculty of Science, Prince of Songkla University, Hat Yai, Songkhla, Thailand. 